\title{
Sağlıklı gönüllülerde, el bilek fleksiyonu elektrofizyolojik çalışmaları etkiliyor mu?
}

\author{
Dr. Pınar DORUK, Dr. Mehmet ADAM, Dr. Berrin LEBLEBici \\ Başkent Üniversitesi Adana Araştırma ve Uygulama Merkezi, FTR Kliniği, Adana
}

ÖZET

AMAÇ: Bu çalışmada sağlıklı gönüllülerde beş dakikalık el bilek fleksiyonunun, karpal tünel sendromu (KTS) tanısında kullanılan sinir iletim çalışmalarından olan, median sinir distal duyu latansı (ddl), 1. parmak median-radial ddl farkı, 4. parmak median-ulnar ddl farkına etkisini araştırmak amaçlanmıştır.

GEREÇ-YÖNTEM: Çalışma 20 kadın ve 5 erkek olmak üzere 25 sağlıklı gönüllü (toplam 46 el) üzerinde yapıldı. Median ddl, 1. parmak median-radial ddl farkı ve 4. parmak median-ulnar ddl farkı incelendi. Beş dakikalık provokatif bilateral $90^{\circ}$ el bilek fleksiyonunu takiben bahsedilen testler tekrarlandı.

BULGULAR: Çalışmaya alınan gönüllülerin yaş ortalaması 32,8土8,4 yıl idi. Ortalama median sinir ddl,

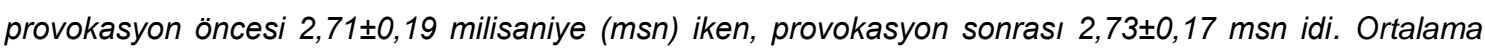
median sinir amplitüdü önce 35,8 $\pm 21,8$ mikrovolt iken, provokasyon sonrası 40,2 $\pm 22,2$ mikrovolt idi. 4.parmak median-ulnar ddl farkı, provokasyon öncesi 0,12 $\pm 0,18 \mathrm{msn}$, provokasyon sonrası 0,15 $\pm 0,19 \mathrm{msn}$ bulundu. 1. parmak median-radial $d d l$ farkı ise önce $0,13 \pm 0,23$ msn iken provokasyon sonrası 0,24 $\pm 0,47$ msn idi.

Provokasyon öncesi ve sonrası median sinir 3. parmak ddl ve amplitüdü, 1. parmak median-radial ddl farkı, 4. parmak median-ulnar ddl farkı değerleri arasında istatistiksel olarak anlamlı fark saptanmadı $(p>0,05)$.

SONUÇ: Sağlıklı gönüllülerde el bilek fleksiyonu şeklindeki provokasyon, elektrofizyolojik çalışmaları etkilememektedir.

Anahtar Sözcükler: El bilek fleksiyonu, sinir iletim hızları, fark testleri

\section{Gíriş}

Karpal tünel sendromu (KTS) en sık görülen ve elektrodiagnostik çalışma amacıyla en fazla refere edilen tuzak nöropatidir(1). Median sinirin bilekte kompresyonu sonucu iskemi, demyelinizasyon, ciddi vakalarda wallerian dejenerasyon ve aksonal kayıp oluşabilir².

Sinir iletim çalışmaları bu hastalarda ayırıcı tanı, teşhis ve derecelendirme açısından önemli olmasına rağmen KTS öntanılı hastaların \% 1025 'sinde normaldir ${ }^{1}$.

Geliş Tarihi: 25.08.2011 Kabul Tarihi:02.12.2011
KTS teşhisinde, en sık median sinir distal motor latansı, distal duyusal latans (ddl), avuç içibilek segmenti iletim hızı ipsilateral ve kontrlateral ulnar siniri ile karşılaştırmalı olarak yapılmaktadır. $\mathrm{Bu}$ testleri normal olan başlangıç düzey KTS olgularında ise 1. parmak median-radial duyu latans farkı, 2. parmak median-ulnar motor latans farkı ve 4. parmak median-ulnar duyu fark testleri çalışılmaktadır. KTS kliniği olan hastalarda el bilek fleksiyonu sonrası fark testleri ve sinir iletim çalışmalarının tekrarlanması tanıya yardımcı olabilir. Ancak provokatif el bilek fleksiyonunun sağlıklı bireylerde sinir iletim çalışmalarına etkisi net değildir. 
Bu çalışmada, KTS öntanılı hastalarda provokasyon amaçlı kullanılan el bilek fleksiyonun, sağlıklı gönüllülerde sinir iletim çalışmaları ve fark testlerine etkisini değerlendirmek amaçlanmıştır.

\section{MATERYAL-METOD}

$\mathrm{Bu}$ çalışma Başkent Üniversitesi Adana Araştırma ve Uygulama Merkezi, FTR Kliniği Elektronöromyografi Laboratuarı'nda gerçekleştirildi. Üst ekstremite nörolojik muayenesi, median ve ulnar sinir duyu ve motor hızları, median sinir motor ve duyu latansları, median sinir avuç içibilek segmenti iletim hızı ve duyusal amplitüdleri normal olan, 20 kadın ve 5 erkek olmak üzere toplam 25 sağlıklı gönüllü (50 el) çalışmaya dahil edildi. Ancak 4 hastanın birer eli, 4. parmak median-ulnar veya 1. parmak median-radial ddl farkından en az birinin median sinir lehine 0,5 msn'den fazla olması nedeniyle çalışma dışı bırakıldı. Çalışma 46 el ile tamamlandı.

Median sinir lezyonu düşündüren belirti ve bulguları (Tinel ve Phalen testi gibi) olanlar, servikal radikülopati, fokal veya generalize nöropati ya da brakial pleksus lezyonu gibi nörolojik problemi olanlar, el bileğine yönelik travma, cerrahi veya enjeksiyon hikayesi olanlar, servikal radikülopati cerrahisi geçirenler, diabetes mellitus ve hipotiroidizm gibi sistemik hastalığı bulunanlar, romatoid artritli hastalar, gebeler çalışmaya alınmadı.

Provokasyon öncesi median sinir ddl'। 3,45 msn'den fazla olanlar, median sinir distal motor latansı 4,0 msn'den fazla olanlar, median sinir motor amplitütü 4,25 mv'dan düşük olanlar ile 4 . parmak median-ulnar ve 1. parmak median-radial ddl farkının en az birinin median sinir lehine 0,5 msn'den fazla olanlar çalışma dışı bırakıldı.

\section{Sinir iletim çalışmaları:}

İletim çalışmaları, gönüllüler hasta muayene masasında otururken, ellerinin rahat bir pozisyonda olması için kucaklarına bir yastık yerleştirerek gerçekleştirildi. Çalışmaların etkilenmemesi için, cilt ısısı düşük olan gönüllülerin elleri ısıtıldıktan sonra sinir iletim çalışmaları yapıldı. Tüm ölçümlerde aktif ve referans elekrot arasındaki mesafe $4 \mathrm{~cm}$ idi. Optimal etkinlik amacıyla toprak elektrod, kayıt elektrod ve stimülatör arasına yerleştirildi.

Median sinir duyu iletimi, yüzük şeklindeki kayıt elektrodu 3. parmağa yerleştirilerek değerlendirildi ve stimülatör kayıt elektrodunun 12 $\mathrm{cm}$ proksimalinde olacak şekilde antidromik olarak yapıldı. Motor sinir iletim çalışması, abduktor pollisis brevis kasından kaydedildi ve kayıt elektrodunun $8 \mathrm{~cm}$ proksimalinden stimülasyon uygulandı. Proksimal uyarı ise antekubital bölgede median sinirin yüzeyelleştiği bölgeden yapıldı. Her iki referans noktası arasındaki mesafe süreye bölünerek median sinirin duysal ve motor ileti hızı saptandı.

1. parmak median-radial ddl farkı ve 4 . parmak median-ulnar ddl farkı eşit mesafeden ve kayıt elektrodu ve stimülatör arasında $8 \mathrm{~cm}$ olacak şekilde kaydedildi.

Daha sonra hastadan her iki el sırtı birbirine değecek şekilde 5 dakikalık zorlu el bilek fleksiyonu yapması istendi. Fleksiyon sonrası median sinir duyusal amplitüdü ve ddl, 1. parmak median- radial ddl farkı ve 4 parmak median- ulnar ddl farkı yeniden çalışıldı. Tüm değerlendirmeler 4 kanallı Medelec Synergy (Oxford Instruments Medical, Surrey, England) cihazı ile aynı hekim tarafından yapıldı.

Verilerin analizi SPSS 17.0 paket programında, paired sampled $\mathrm{T}$ test ile yapıldı. $p<0,05$ değeri istatistiksel olarak anlamlı kabul edildi.

Çalışmaya katılan tüm sağlıklı gönüllülerden yazılı onam alındı.

\section{BULGULAR:}

Çalışma populasyonunun yaş ortalaması $32,8 \pm 8,4$ (20-49) idi. Sinir iletim çalışması ve fark testlerine ait sonuçlar tablo 1'de gösterilmektedir. Provokasyon öncesi ve sonrası ortalama median sinir ddl, median sinir amplitüdü, 1. parmak median- radial ddl farkı, 4. parmak median-ulnar ddl 
farkları arasında istatistiksel olarak anlamlı fark saptanmadı $(p>0.05)$.

Tablo 1: Provokasyon öncesi ve sonrası sinir iletim çalışmaları sonuçları

\begin{tabular}{|l|l|l|l|}
\hline & Provokasyon öncesi & Provokasyon sonrası & p \\
\hline Median sinir ddl (ortalama $\pm S S)(m s n)$ & $2,71 \pm 0,19$ & $2,73 \pm 0,17$ & $>0,05$ \\
\hline Median sinir amplitüd (ortalama $\pm S S)(\mu \mathrm{V})$ & $35,8 \pm 21,8$ & $40,2 \pm 22,2$ & $>0,05$ \\
\hline 1. parmak median -radial ddl farkı (ortalama $\pm S S)$ & $0,13 \pm 0,23$ & $0,24 \pm 0,47$ & $>0,05$ \\
\hline 4. parmak median -ulnar ddl farkı (ortalama $\pm S S)$ & $0,12 \pm 0,18$ & $0,15 \pm 0,19$ & $>0,05$ \\
\hline
\end{tabular}

ddl: duysal distal latans, SS: Standart sapma

\section{TARTIŞMA}

En sık görülen üst ekstremite kompresyon nöropati olan karpal tünel sendromu (KTS) tüm tuzak nöropatilerin \%90'ını meydana getirmektedir $^{3}$. Bu sendromda median sinir innervasyonu olan bölgelerde geceleri artan parestezi, ağrı, hissizlik görülebilir ${ }^{1}$. KTS'de tanı koydurucu standart kriterler üzerinde hala tartışmalar bulunmaktadır ${ }^{4}$.

KTS nin klinik ya da elektrofizyolojik bir tanı olması konusunda belirli bir görüş birliği yoktur. KTS tanısının sadece kesin klinik hikaye ve fiziksel muayeneye dayandırılması gerektiğini düşünen klinisyenler olmakla birlikte, KTS tanısının sadece subjektif semptom ve bulgulara dayandırımasının ayırıcı tanı açısından problem oluşturabileceğini de düşünenler vardır. Elektrofizyolojik çalışmaların KTS öntanılı hastalarda tanı koyma potansiyeline sahip olduğuna inanılmaktadır ${ }^{5}$.

Sinir ileti çalışmaları KTS tanısında oldukça özgül bir metod olmasına rağmen, bazı hastalarda bu çalışmalar normal olabilmektedir, ${ }^{6,7}$. Hatta, KTS semptom ve bulguları bulunan hastaların \%13-27'si kadar yüksek oranda normal bulunabildiği bildirilmektedir $^{8}$. Normal elektrofizyolojik çalışmaların KTS tanısını dışlamaması gerektiği ifade edilmiştir 9 .
KTS ön tanılı hastalarda elektrofizyolojik çalışmaların hassasiyetini arttırmak için çeşitli yöntemler kullanılagelmiştir. Median sinir ile ipsilateral ulnar yada radial sinir duyu latanslarının karşılaştırıldığı fark çalışmaları daha sensitif olmasına ve tanıda \%10 oranında ek katkı sağlamasına ${ }^{10,11}$ rağmen, bu sonuçların da yanlış pozitif olabileceği bildirilmiştir ${ }^{9}$.

Sinir iletim çalışmaları ve fark testleri normal olan KTS öntanılı hastalarda izlenebilecek yöntemlerden bir tanesi el bilek fleksiyonu sonrası sinir iletim çalışmalarının yeniden uygulanmasıdır. Dunnan ve ark. 68 kontrol ve 19 KTS hastasında el bilek fleksiyonunun KTS teşhisinde ek yarar sağlamayacağını bildirmişlerdir ${ }^{12}$. Bu çalışmanın aksine, Emad ve $\operatorname{ark}^{13}, 20$ şüpheli KTS hastasının ve 20 sağlıklı eli değerlendirdikleri çalışmalarında, 5 dakikalık el bilek kompresyonu sonrasında şüpheli KTS hastalarında median sinir ddl ve el bilek bölgesi sinir iletim zamanında etkilenme bulmuşlardır. Keza, Hansson ve ark ${ }^{14}$, klinik olarak 10 KTS tanılı hasta, 20 şüpheli KTS ve 10 normal bireyi değerlendirdikleri çalışmalarında, el bilek fleksiyonu sırasında median sinir duyusal aksiyon potensiyallerinin KTS tanısında hassasiyeti ve özgüllüğü arttırabileceğini savunmuşlardır. Bu çalışmanın kontrol grubundaki 10 kişiden 8 
tanesinde el bilek fleksiyonu ile median sinir duysal aksiyon potansiyel amplitüdünde düşme olduğu, el bilek fleksiyonunun median sinirde duysal iletiyi etkilediği görülmüştür.

Nispeten daha fazla olgunun alındığı çalışmamızda el bilek fleksiyonu öncesi ve sonrası median sinir amplitüdü ve ddl, median sinir ile radial ve ulnar sinir distal latans farkları arasında fark yoktu. Sağlıklı bireylerde el bilek fleksiyonunun median sinirde kanlanma azlığı, iskemi gibi fizyolojik değişikliklere neden olmadığı ya da bu değişikliklerin sinir ileti çalışmalarını etkileyebilecek düzeyde olmadığı düşünülebilir. Fizyolojik değişikliklere neden olsa bile, provokasyon sonrası el bileğinin nötral pozisyona dönülmesi oluşan değişikliklerin hızla normale dönmesine ve düzelmesine sebebiyet verebilir.

Çalışmamızda sağlıklı gönüllülerde el bilek fleksiyon sonrası sinir iletim çalışmalarının etkilenmesinin bulunması halinde, klinik olarak şüpheli KTS hastalarında rutin ENMG çalışmalarına ilaveten kullanılabilecek bir çalışmanın kapısının aralanmasına neden olabilecekti. Sonuç olarak, KTS tanısında kullanılan sinir ileti çalışmaları ve fark testleri, sağlıklı bireylerde el bilek fleksiyonundan etkilenmediği çalışmamızda gösterilmiştir.

\section{SUMMARY}

Does the wrist flexion affect on the electrophysiological studies in the healthy voluntaries?

PURPOSE: The aim of this study is to evaluate the effects on the median nerve distal sensory latencies (dsl), first digit median vs. radial sensory latency difference, fourth digit median vs. ulnar sensory latency difference which are repeated after performing the provocative wrist flexion for about five minutes in the healhty voluntaries.

MATERIALS-METHODS: In this study, 20 women, 5 men voluntaries (total 46 hands) were investigated. The median nerve dsl, the first digit median vs. radial sensory latency differences, the fourth digit median vs. ulnar sensory latency differences were recorded. After performing the provocative wrist flexion (bilaterally 90 degree) for about five minutes, above mentioned studies were repeated.

RESULTS: The mean age of the voluntaries in the study was $32.8 \pm 8.4$ years. Before and after provocation, the mean dsl of median nerve was $2.71 \pm 0.19 \mathrm{msc}$, and $2.73 \pm 0.17 \mathrm{msc}$, and the mean amplitude of median nerve was $35.8 \pm 21.8$, and $40.2 \pm 22.2$ microvolt, the mean of fourth digit median vs. ulnar sensory latency differences was $0.12 \pm 0.18 \mathrm{msc}$, and $0.15 \pm 0.19 \mathrm{msc}$, the mean of first digit median vs. radial sensory latency differences was $0.13 \pm 0.23 \mathrm{msc}$ and $0.24 \pm 0.47$ msc, respectively.

Statistically, there were no significance between before and after the provocation at the mean value of amplitude and dsl, the median vs. radial first digit and the median vs. ulnar fourth digit sensory latency differences measurements ( $p>0.05)$.

CONCLUSION: The provocative wrist flexion in the healthy voluntaries has no effect on the electrophysiological studies.

Key Words: Wrist flexion, nerve conduction study, difference tests.

\section{KAYNAKLAR}

1. Preston DC, Shapiro B. Electromyography and neuromuscular disorders, clinical-electrophysiologic correlations. 2nd ed. Philadelphia: Elsevier, 2005; 255-79.

2. Werner RA, Andary M. Carpal tunnel syndrome: pathophysiology and clinical neurophysiology. Clin Neurophysiol 2002;113(9):1373-81.

3. Aroori S, Spence RA. Carpal tunnel syndrome. Ulster Med J 2008;77(1):6-17.

4. Gomes I, Becker J, Ehlers JA, Nora DB. Prediction of the neurophysiological diagnosis of carpal tunnel syndrome from the demographic and clinical data. Clin Neurophysiol 2006;117(5):964-71.

5. Jablecki CK, Andary MT, So YT, Wilkins DE, Williams $\mathrm{FH}$. Literature review of the usefulness of nerve conduction studies and electromyography for the evaluation of patients with carpal tunnel syndrome. Muscle Nerve 1993;16(12):1392-1414. 
6. Nora DB, Becker J, Ehlers JA, Gomes I. Clinical features of 1039 patients with neurophysiological diagnosis of carpal tunnel syndrome. Clin Neurol Neurosurg 2004;107(1):64-9.

7. Ortiz-Corredor F, Calambas N, Mendoza-Pulido C, Galeano J, Díaz- Ruíz J, Delgado O. Factor analysis of Carpal Tunnel Syndrome Questionnaire in relation to nerve conduction studies. Clin Neurophysiol 2011 March 29 (Epub ahead of print).

8. Bodofsky EB, Wu KD, Campellone JV, Greenberg WM, Tomaio AC. A sensitive new median-ulnar technique for diagnosing mild carpal tunnel syndrome. Electromyogr Clin Neurophysiol. 2005;45(3):139-44

9. Atroshi I, Gummesson C, Johnsson R, Ornstein E, Ranstam J, Rosen I. Prevalence of carpal tunnel syndrome in general population. JAMA 1999;282(2):153-8.

10. Sandin KJ, Asch SM, Jablecki CK, Kilmer DD, Nuckols TK; Carpal Tunnel Quality Group. Clinical quality measures for electrodiagnosis in suspected carpal tunnel syndrome. Muscle Nerve 2010;41(4):444-52.

11. Chang MH, Liu LH, Lee YC, Wei SJ, Chiang HL, Hsieh PF. Comparison of sensitivity of transcarpal median motor conduction velocity and conventional conduction techniques in electro diagnosis of carpal tunnel syndrome. Clin Neurophysiol 2006;117(5):984-91.

12. Dunnan JB, Waylonis GW. Wrist flexion as an adjunct to the diagnosis of carpal tunnel syndrome. Arch Phys Med Rehabil 1991;72(3):211-3 (abs).

13. Emad MR, Najafi SH, Sepehrian MH. The effect of provocative tests on electrodiagnosis criteria in clinical carpal tunnel syndrome. J Electromyogr Kinesiol 2009;19(6):1061-63.

14. Hansson S, Nilsson BY. Median sensory nerve conduction block during wrist flexion in the carpal tunnel syndrome. Electromyogr Clin Neurophysiol 1995;35(2):99-105.

\section{Yazışma Adresi:}

Dr. Pınar Doruk

Başkent Üniversitesi Adana Araştırma ve

Uygulama Merkezi, FTR Kliniği

Yüreğir/ADANA

Tel: 03223444444

e-posta: doruk.pinar@gmail.com 